\title{
HIDROCEFALIA DE PRESSÃO NORMAL: AVALIAÇÃO DE CINCO ANOS DE EXPERIÊNCIA E REVISÃO DE LITERATURA
}

\author{
NORMAL-PRESSURE HYDROCEPHALUS: EVALUATION OF \\ FIVE-YEAR EXPERIENCE AND LITERATURE REVIEW
}

Luciano Melato'; Marcelo E. Bigal² \& José G. Speciali ${ }^{3}$

\begin{abstract}
${ }^{1}$ Médico Residente em Clínica Médica; ${ }^{2}$ Mestre em Neurologia; ${ }^{3}$ Docente. Faculdade de Medicina de Ribeirão Preto, Universidade de São Paulo.

Correspondência: Prof. Dr. José G. Speciali. Faculdade de Medicina de Ribeirão Preto. Departamento de Neurologia. Av. Bandeirantes, 3900. 14049-900. Ribeirão Preto (SP) Brasil.
\end{abstract}

MELATO L; BIGAL ME \& SPECIALI JG. Hidrocefalia de pressão normal: avaliação de cinco anos de experiência e revisão de literatura. Medicina, Ribeirão Preto, 33: 499-505, out./dez. 2000.

RESUMO: Hidrocefalia com pressão normal (HPN) é uma síndrome caracterizada por apraxia de marcha, demência e incontinência urinária, sendo uma das causas tratáveis de demência. O presente estudo avaliou os dados clínicos, laboratoriais e o tratamento dos pacientes com HPN, atendidos no período de 1992 a 1997, no Hospital das Clínicas da Faculdade de Medicina de Ribeirão Preto. A hipótese diagnóstica de HPN foi feita em 56 casos, tendo sido confirmada em 30. Distúrbios motores foram verificados em $100 \%$ dos casos, alterações cognitivas em $83,3 \%$ e distúrbios esfincterianos em $63,3 \%$. A tríade ocorreu em $53,3 \%$ dos casos. A tomografia computadorizada mostrou hidrocefalia em $96,7 \%$ dos pacientes, sendo que, em $40 \%$, havia evidência ainda de isquemia cerebral. Os pacientes foram submetidos a dois tipos de tratamento: punções liquóricas de repetição e instalação de derivação liquórica. No primeiro caso, observou-se melhora em 53,3\%. Já, no caso da instalação de derivação liquórica, observou-se melhora em $63,1 \%$. Conclui-se que HPN é uma síndrome que não pode ser considerada incomum, devendo, sempre, ser lembrada como diagnóstico diferencial de demências e distúrbios de marcha do idoso, por tratar-se de patologia potencialmente tratável.

UNITERMOS: Hidrocefalia com Pressão Normal.

\section{INTRODUÇÃO}

O reconhecimento da hidrocefalia de pressão normal (HPN), como causa potencialmente tratável de demência, tem recebido atenção crescente. Essa síndrome, enquanto entidade nosológica distinta, foi primeiramente reconhecida por Hakim ${ }^{(1)}$ e Adams et al. ${ }^{(2)}$, na década de 60. Caracteriza-se, tipicamente, por um desenvolvimento gradual (ao longo de semanas ou meses), de alterações na marcha, associada a graus variáveis de declínio intelectual, que progride insidiosamente para níveis mais avançados de demên- cia e incontinência urinária ${ }^{(3,4)}$. Essa tríade é considerada classicamente como o marcador clínico dessa doença, embora não esteja presente em todos os casos, nem seja específica. Pacientes, geralmente, não apresentam cefaléia ou outros sinais de hipertensão intracraniana. Punções liquóricas, usualmente, demonstram pressão inicial normal.

O diagnóstico encontra suporte nos seguintes achados:

1) punções liquóricas com pressão de abertura normal;

2) hidrocefalia confirmada por exames de neuroima- 
gem, como tomografia computadorizada ou ressonância magnética.

3) cisternografia radioisotópica, demonstrando alterações no fluxo liquórico;

4) testes funcionais, como a resposta temporária à retirada de grandes quantidades de líquido cefalorraqueano. No entanto, é consenso considerar-se tal síndrome como de diagnóstico eminentemente clínico/ neurológico ${ }^{(5)}$.

O presente trabalho tem como objetivo avaliar os dados clínicos dos pacientes com HPN, atendidos no Hospital das Clínicas de Ribeirão Preto (HC), no período de 1992 a 1997, bem como relacioná-los com os exames de imagem e tratamento instituído.

\section{MATERIAL E MÉTODOS}

Foram revisados os prontuários de 56 pacientes atendidos no Hospital das Clínicas da Faculdade de Medicina de Ribeirão Preto - USP, no período de 1992 a 1997, com suspeita diagnóstica de HPN. Esse hospital é unidade de referência terciária em neurologia para toda a região. Desses, 30 tiveram o diagnóstico confirmado pelo médico assistente. Para tanto, utilizaramse critérios clínicos sugestivos, exames de imagens, ou cisternografia, compatíveis e respostas favoráveis ao teste com punções liquóricas. Foram avaliados os sinais e sintomas apresentados, dados demográficos (idade, sexo, raça), tempo e modo de evolução, antecedentes pessoais, hipótese diagnóstica inicial formulada, dados dos exames laboratoriais e resposta ao tratamento. Compararam-se as duas modalidades terapêuticas utilizadas, punções liquóricas de repetição e instalação de derivação liquórica.

\section{RESULTADOS}

No período estudado, HPN foi aventada como hipótese diagnóstica em 56 casos atendidos, tendo sido confirmada em $30(53,6 \%)$ deles. Os demais casos foram de patologias classicamente consideradas como diagnóstico diferencial de HPN e estão listadas na Tabela I.

Dos 30 casos cujo diagnóstico final foi HPN, em apenas $14(46,7 \%)$ ela foi a hipótese diagnóstica inicial. As outras hipóteses diagnósticas consideradas estão listadas na Tabela II.
Dentre os casos de HPN, 23 (76,7\%) eram do sexo masculino e $28(93,3 \%)$ eram de raça branca. Apenas um paciente $(3,3 \%)$ apresentava idade menor que 50 anos, sendo que $26(86,7 \%)$ pacientes tinham mais que 60 anos.

O tempo decorrido entre o aparecimento da sintomatologia e o diagnóstico está apresentado na Tabela III.

\begin{tabular}{|lc|}
\hline $\begin{array}{l}\text { Tabela I - Diagnósticos dos casos não confirmados } \\
\text { como HPN. }\end{array}$ & Número de Casos \\
\hline Diagnóstico Final & 10 \\
\hline Demência por múltiplos infartos & 4 \\
Doença de Alzheimer & 4 \\
Hidrocefalia hipertensiva & 3 \\
Doença de Biswanger & 2 \\
Psicoses & 1 \\
Neurolues & 2 \\
\hline Sem confirmação diagnóstica & \\
\hline
\end{tabular}

Tabela II - Diagnósticos, na primeira consulta, para os pacientes com HPN.

\begin{tabular}{lrr} 
Diagnóstico Inicial $^{*}$ & $n^{*}(\%)$ \\
\hline Hidrocefalia de pressão normal & $14(46,7)$ \\
Demência de etiologia a esclarecer & 6 & $(20)$ \\
Síndrome parkinsioniana/extrapiramidal & 6 & $(20)$ \\
AVC / AIT & $4(13,3)$ \\
Síndrome frontal de etiologia a esclarecer & $2(6,7)$ \\
Convulsões de etiologia a esclarecer & $2(6,7)$ \\
Neurocisticercose & $1(3,3)$ \\
Síndrome piramidal de etiologia a esclarecer & $1(3,3)$ \\
Hidrocefalia obstrutiva & $1(3,3)$ \\
Impregnação por neurolépticos & $1(3,3)$ \\
Degeneração cerebelar & $1(3,3)$
\end{tabular}

Legenda: AVC: acidente vascular cerebral; AIT: ataque isquêmico transitório. * Alguns pacientes tiveram mais de um diagnóstico sugerido. 


\begin{tabular}{|lc|}
\hline $\begin{array}{l}\text { Tabela III - Tempo decorrido entre o início da sin- } \\
\text { tomatologia e o diagnóstico }\end{array}$ \\
\hline Tempo de Evolução & $\mathrm{n}(\%)$ \\
\hline Até 6 meses & $14(46,7)$ \\
6 - 12 meses & $5(16,7)$ \\
1 - 2 anos & $1(3,2)$ \\
2 - 3 anos & $5(16,7)$ \\
$>3$ anos & $5(16,7)$ \\
\hline
\end{tabular}

Dos casos estudados, $28(93,3 \%)$ apresentaram início insidioso e evolução progressiva. Um caso apresentou início súbito com crise convulsiva, em paciente previamente descrito como hígido, e um caso apresentou evolução descrita como intermitente, com períodos de melhoria e exacerbação dos sintomas.

A Tabela IV apresenta os principais sintomas e sinais encontrados. A tríade clássica (distúrbios da marcha, cognitivos e esfincterianos) foi verificada em $16(53,3 \%)$ dos pacientes.

Dentre os achados de piramidalismo, o mais comum foi a hiperreflexia, seguida pela marcha espástica. Sinal de Babinski foi verificado em dois casos, assim como sinal de Hoffman. Marcha bradicinética foi o sinal extrapiramidal mais freqüente, seguida de rigidez postural. O distúrbio da marcha mais comum após a bradicinesia foi a apraxia.

Outros sinais e sintomas menos frequientes foram: sinais focais (dois casos), sintomas bulbares (dois casos), ataxia cerebelar (dois casos), tremor de repouso (dois casos), movimentos coréicos de membros superiores (um caso) e tonturas (um caso).

Os antecedentes pessoais mais comuns, nos pacientes com HPN, foram os seguintes: hipertensão arterial (40\% pacientes), tabagismo (26,7\%), etilismo $(23,3 \%)$ e diabetes melito $(6,7 \%)$. Antecedentes de acometimento estrutural prévio do sistema nervoso central ocorreu em 11 casos $(32,7 \%)$, sendo quatro de acidente vascular cerebral, três de traumatismo cranioencefálico, um de hemorragia subaracnóidea, um de neuro- cirurgia, um de neurocisticercose e um de neurolues. O exame subsidiário mais solicitado foi a tomografia computadorizada (CT), realizado em todos os casos. Os achados estão mostrados na Tabela V.

Chama a atenção a elevada percentagem de infartos encontrada (40\%).

Cisternografia foi realizada em 10 pacientes. $\mathrm{O}$ resultado foi sugestivo de HPN em 8, inconclusivo em um e demonstrou atrofia cerebral em um. Registro contínuo da pressão foi obtido em dois pacientes.

Com a confirmação diagnóstica, os pacientes foram submetidos a dois tipos de procedimento: punções liquóricas de repetição ou instalação de derivação liquórica. O primeiro procedimento foi realizado em 15 pacientes. Os demais receberam instalação de derivação sendo que, em 10 casos, essa derivação foi lomboperitoneal e, em nove foi ventriculoperitoneal. As médias de pressão inicial e final, quantidade de líquido cefalorraqueano (LCR) retirada e número de punções realizadas estão expostos na Tabela V. A resposta ao tratamento (clínico ou cirúrgico) está apresentada no gráfico 1 . Um total de $53 \%$ dos pacientes

\begin{tabular}{|c|c|}
\hline SINTOMAS / SINAIS & $N(\%)$ \\
\hline Distúrbios motores & $30(100)$ \\
\hline Alterações da marcha & $29(96,7)$ \\
\hline Sinais piramidais & $14(46,7)$ \\
\hline Sinais extrapiramidais & $10(33,3)$ \\
\hline Reflexos patológicos (grasping e palmomentoniano) & $8(26,7)$ \\
\hline Sintomas cognitivos & $25(83,3)$ \\
\hline Déficit de memória & $12(40)$ \\
\hline Alteração de personalidade/comportamento & $12(40)$ \\
\hline Confusão mental & $4(13,3)$ \\
\hline Rebaixamento da consciência & $5(16,7)$ \\
\hline Distúrbios esfincterianos & $19(63,3)$ \\
\hline Incontinência urinária & $19(63,3)$ \\
\hline Incontinência fecal & $3(10)$ \\
\hline Crises convulsivas & $3(10)$ \\
\hline Cefaléia & $3(10)$ \\
\hline Outros & $10(33,3)$ \\
\hline
\end{tabular}

n: valor absoluto; \% valor relativo. 


\begin{tabular}{|lc|}
\hline $\begin{array}{l}\text { Tabela V - Achados tomográficos em pacientes } \\
\text { com HPN. }\end{array}$ \\
\hline Tomografia computadorizada & $N(\%)$ \\
\hline Hidrocefalia & $29(96,7)$ \\
Atrofia cerebral difusa & $11(36,7)$ \\
Calcificações & $4(13,3)$ \\
Atrofia cortical & $3(10)$ \\
Atrofia cerebelar & $6(20)$ \\
Infartos cerebrais & $12(40)$ \\
Hipodensidade periventricular & $2(6,7)$ \\
Edema transependimário & $1(3,3)$ \\
\hline
\end{tabular}

Tabela VI - Média dos achados liquóricos nos 14 pacientes submetidos a tratamento clínico.

\begin{tabular}{|lc|}
\hline \multicolumn{2}{|c|}{ Características do LCR } \\
\hline Pressão inicial & $12,8 \mathrm{~cm} \mathrm{H} \mathrm{C}_{2}$ \\
Pressão final & $4,4 \mathrm{~cm} \mathrm{H} \mathrm{O}_{2}$ \\
Volume retirado & $21,6 \mathrm{ml}$ \\
Número de punções & 3,1 \\
\hline
\end{tabular}

submetidos a punções de repetição e $63,3 \%$ dos pacientes que receberam derivação liquórica obtiveram algum grau de melhora, sendo que a marcha foi a mais beneficiada.

Dos pacientes submetidos a cirurgia, em três foi realizada previamente a punção liquórica. Todos apresentaram melhora clínica à retirada do LCR e boa evolução após a cirurgia. Os demais foram submetidos a cirurgia sem a realização desse teste funcional.

\section{DISCUSSÃO}

Nos seis anos estudados (1992 - 1997), a hipótese de hidrocefalia de pressão normal foi sugerida em 56 pacientes atendidos no HC. Desses, 30 tiveram diagnóstico confirmado pelos critérios expostos. Em 26 pacientes, o diagnóstico inicial de HPN não foi confirmado posteriormente. Por outro lado, daqueles 30 pacientes, em 16 deles não foi aventado o diagnóstico inicial de HPN, mas o seguimento confirmou tal diagnóstico. Dados de literatura mostram que HPN é o diagnóstico final em cerca de $5 \%$ dos pacientes com demência ${ }^{(6,7)}$, justificando os principais diagnósticos iniciais aventados (demência de etiologia a esclarecer, AVC/AIT, síndrome frontal, neurocisticercose). É síndrome, no entanto, que deve sempre ser lembrada, para que o diagnóstico clinico possa ser feito.

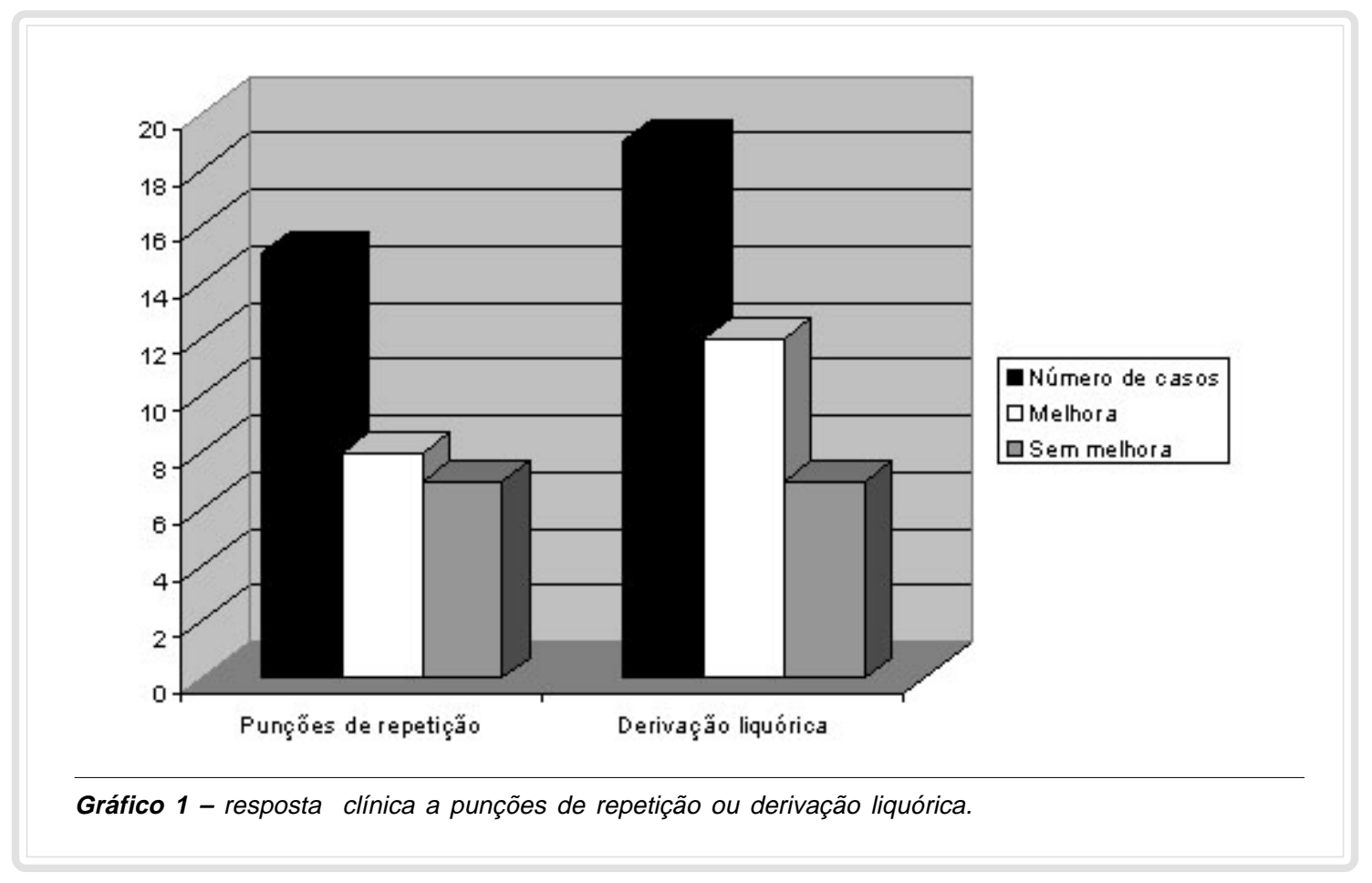


Em 63,4\% dos casos, o diagnóstico foi feito com menos de um ano do aparecimento dos sintomas e os pacientes tinham mais que 60 anos de idade. Em nossa casuística houve predomínio de pacientes do sexo masculino e brancos.

A fisiopatologia presumida da HPN implica um aumento da resistência ao fluxo liquórico, a diminuição da sua absorção ou ambos. Estudos recentes demonstram que, em pessoas normais, a resistência ao fluxo liquórico aumenta com a idade ${ }^{(8)}$. Além disso, o número de casos idiopáticos é menor em pacientes mais jovens ${ }^{(9)}$.

Desde os trabalhos clássicos de Hakim ${ }^{(1)}$, delineou-se, como quadro clínico típico da HPN, a tríade, distúrbios da marcha, incontinência urinária e demência. Deve-se lembrar, e nossos dados são coincidentes com isso, que a tríade não ocorre em todos os casos nem é específica dessa doença. Também ficou demonstrado que a ordem do aparecimento dos sintomas, geralmente, era a seguinte: o distúrbio de marcha, precedendo sinais clínicos de demência ${ }^{(1,2)}$. Considera-se, atualmente, que pacientes com demência sem distúrbio da marcha usualmente não apresentam hidrocefalia sintomática ${ }^{(4,10)}$. Nossos dados são concordantes com tal hipótese. A maioria dos pacientes apresentavam distúrbios motores e, em freqüência menor, distúrbios cognitivos e esfinctéricos. O distúrbio inicial mais freqüente da marcha é o alentecimento global, com tendência a queda e perda dos movimentos $\operatorname{associados}^{(4,10)}$. Isso foi verificado também em nossos pacientes. A seguir, encontramos apraxia de marcha, o que também coincide com achados de literatura $^{(11)}$. Em relação ao quadro cognitivo, o usual é o padrão de demência subcortical, fato por nós também verificado. Há, na literatura, descrição de apresentações atípicas ${ }^{(12)}$, incluíndo hipotermia, "drop attacks", epilepsia, dor em membros ou no dorso. Em nossa casuística, observamos algumas dessas apresentações, como: epilepsia, movimentos coréicos de membros superiores, dor em membros inferiores, tonturas, etc. Tais casos poderiam, eventualmente, enquadrar-se em alguma etiologia sintomática de HPN, não discriminada pelos métodos diagnósticos usados. Os antecedentes pessoais não diferem significativamente dos encontrados em idosos saudáveis.

Tomografia computadorizada é considerada um método importante no processo diagnóstico, tendo sido realizada em todos os nossos pacientes. Pode demonstrar uma patologia primária como causa, ou alterações sugestivas de lesões prévias. Quando nenhuma patologia é revelada, sinais prognósticos, considerados favoráveis, incluem hipodensidade periventricular, aumento do quarto ventrículo e apagamento dos sulcos corticais ${ }^{(13)}$. Esse último achado oferece melhor prognóstico à colocação da derivação. Hidrocefalia com atrofia e pressão normal, geralmente, indica tratar-se de hidrocefalia ex-vácuo, com pior prognóstico ao tratamento. Chama a atenção, nos nossos dados, a presença de alterações isquêmicas em $40 \%$ dos exames. Eventualmente, tais alterações poderiam dificultar o fluxo liquórico, contribuindo para a sintomatologia ou, ao contrário, representar sobreposição clínica entre a HPN e a demência por múltiplos infartos ${ }^{(14,15)}$.

Atualmente, considera-se a monitorização da pressão intracraniana como parte do processo diagnóstico. Estima-se que melhores resultados à derivação teriam pressão liquórica na faixa superior da normalidade $^{(16)}$. Tal método permitiria ainda demonstrar o aumento transitório da pressão intracraniana ao longo do dia. No período pesquisado, tal procedimento não era feito com freqüência em nosso hospital. No entanto, a maioria de nossos pacientes com bons resultados ao tratamento apresentava pressão liquórica no limite superior da normalidade. Estudos recentes têm demonstrado que esse método seria valioso no diagnóstico diferencial entre HPN e demência com hidrocefalia ex-vácuo, problema clínico dos maiores ${ }^{(17)}$. A cisternografia, geralmente, demonstra pouca passagem do marcador para a convexidade, fato encontrado na maioria de nossos pacientes com os quais se utilizou esse método. Existem estudos atuais que tentam mensurar o fluxo liquórico por meio da ressonância magnética, com excelentes resultados, o que tenderia a limitar o uso da cisternografia ${ }^{(18)}$. Alguns autores consideram que a cisternografia já se encontra totalmente superada em função da ressonância nuclear magnética, capaz de mensurar a dinâmica liquórica. Esse exame é capaz de oferecer informações quantitativas sobre o fluxo liquórico aumentado, que se verifica, entre outros, no aqueduto de Sylvius de pacientes com HPN, em especial ao se utilizarem técnicas de alta resolução em cortes axiais. Mostra ainda que, a despeito de a pressão liquórica encontrar-se normal, o pulso de pressão liquórica que acompanha o ciclo cardíaco, em pacientes com HPN, encontra-se aumentado de seis a oito vezes em relação aos valores normais ${ }^{(19)}$.

Tradicionalmente, realiza-se o teste de retirada de LCR ("tap test") como preditivo da resposta à instalação da derivação liquórica ${ }^{(20)}$. No entanto, existe tendência atual de se considerar tal teste apenas quan- 
do positivo. Ou seja, resposta negativa não contraindicaria a cirurgia. Malm et al. ${ }^{(17)}$ consideram, inclusive, que tal teste não teria nenhum caráter preditivo. Estudos recentes vêm testando a ressonância magnética como subsidiária, na inferência prognóstica. Trabalho com 20 pacientes com HPN, aqueles que apresentavam sinais de hiperfluxo liquórico no nível do terceiro ventrículo, responderam melhor à derivação ventriculoperitoneal. Quanto menor o fluxo, pior a resposta $^{(21)}$. Assim, há tendência em se considerar esses sinais, detectados na ressonância magnética, como preditores da resposta cirúrgica.

A derivação liquórica é o método padrão de tratamento. Geralmente, o distúrbio da marcha é o mais beneficiado por esse procedimento, sendo que o quadro demencial é menos influenciado ${ }^{(20)}$, fato também observado em nossos pacientes. Dados de literatura demonstram que a melhora no estado mental pode ser percebida apenas após alguns meses, enquanto, em outros pacientes, ocorre apenas melhora transitória pós-operatória. Há aparente paradoxo no fato de que alguns pacientes apresentam melhora a despeito dos ventrículos cerebrais permanecerem aumentados após a cirurgia, embora a regra seja que a redução dos ventrículos correlacione-se com o grau de melhora observada $^{(22)}$.

Conclui-se que HPN é síndrome que não pode ser considerada incomum, devendo, sempre, ser lembrada como diagnóstico diferencial de demências e distúrbios de marcha no idoso. A avaliação clínica é fundamental, bem como a correta propedêutica laboratorial, por tratar-se de patologia tratável.

MELATO I; BIGAL ME \& SPECIALI JG. Normal-pressure hydrocephalus: evaluation of five-year experience and literature review. Medicina, Ribeirão Preto, 33: 499-505, oct./dec. 2000.

ABSTRACTS: Normal-pressure hydrocephalus (NPH) is characterized by the clinical triad of gait apraxia, dementia and urinary incontinence, being one of the reversible causes of dementia. The present study evaluated clinical and laboratory data and the patients' treatment with this disease assisted from 1992 to 1997 in the University Hospital of Ribeirão Preto. The hypothesis of NPH was made in 56 cases, having been confirmed in 30. Disturbances of the motor's function were verified in $100 \%$ of the cases, cognitive alterations in $83,3 \%$ and sphincter disorders in $63,3 \%$. The triad was seen in $53,3 \%$ of the cases. CT scan showed hydrocephalus in $96,7 \%$ of the patients, and in $40 \%$ there was still evidence of ischaemic disorders. Patients were submitted to two kinds of treatment: sucessive spinal fluid drainage or ventricular shunts. In the first case improvement was observed in $53,3 \%$, against $63,1 \%$ in shunting cases. In conclusion, NPH is a syndrome that is not uncommon, and should always be reminded as a differential diagnosis of dementia and gait disturbances, being a potentially treatable disease .

UNITERMS: Hydrocephalus, Normal Pressure..

\section{REFERÊNCIAS BIBLIOGRÁFICAS}

1 - HAKIM S. Algunas observaciones sobre la presion del LCR. Sindrome hidrocefalico en el adulto con "presion normal" del LCR. Thesis 957, Javeriana University School of Medicine, Bogotá, Colombia. p. 1-142, 1964.

2 - ADAMS RD; STEWART K \& HOPKINS HDI. Symptomatic occult hydrocephalus with "normal" cerebrospinal-fluid pressure. N Engl J Med 273:117-126, 1965.

3 - FISCHMAN RA. Normal pressure hydrocephalus and arthritis. N Engl J Med 312: 1255-1256, 1985.

4 - FISHER CM. Hydrocephalus as a cause of disturbances of gait in the elderly. Neurology 32: 1358-1363, 1982.
5 - BLACK PM. The normal pressure hydrocephalus syndrome. In: SCOTT R, ed. Hydrocephalus. concepts in neurosurgery. Willians \& Wilkins, Baltimore, v. 3, p.109-114, 1990.

6 - KARTZMANN R. Normal pressure hydrocephalus. Contemp Neurol 15: 69-92. 1977.

7 - SMITH SS \& KILOH LG. The investigation of dementia: results in 200 consecutive admissions. Lancet 1: 824-827, 1981.

8 - ALBECK MJ \& SKAK C. Age dependency of resistane to cerebroespinal fluid outflow. J Neurosurg 89: 275-278, 1998.

9 - BRET P \& CHAZAL J. Chronic ("normal pressure") hydrocephalus in chidhhod and adolescence. A review of 16 cases and reappraisal of the syndrome. Child Nerv Syst 11: 687$691,1995$. 
10 - FISHER CM. The clinical picture in occult hydrocephalus. Clin Neurosurg 24: 270-284, 1977.

11 - ESTANOL BV. Gait apraxia in communicanting hydrocephalus. J Neurol Neurosurg Psychiatry 44: 305308, 1981.

12 - YUSUF SW; BOOTH SA \& MISHRA RM. Falls and urinary incontinence in a 66-year-old woman. Lancet 347: 1738, 1996.

13 - JACOBS L \& KINKEL W. Computadorized axial transverse tomography in normal pressure hydrocephalus. Neurology 26: 501-507, 1980.

14 - TAYLOR RF \& BEARD MV. Normal pressure hydrocephalus and deep white matter stroke: a case report. Arch Phys Med Rehabil 78: 1015-1018, 1997.

15 - DAVIS PC; MIRRA SS \& ALAZRAKI N. The brain in older person with and without dementia: findings on MR, PET and SPECT images. Am J Radiol 162:1267-1278, 1994.

16 - BORGENSEN SE. Conductance to outflow of CSF in normal pressure hydrocephalus. Acta Neurochir 71: 1-45, 1984.

17 - MALM J \& KRISTENSEN B. The predictive value of cerebrospinal fluid dynamic tests in patients with the idiopatic adult hydrocephalus syndrome. Arch Neurol 52: 783-789, 1995.
18 - BRADLEY WG \& SCALZO D. Normal pressure hydrocephalus: evaluation with cerebrospinal fluid flow measurements at MR imaging. Radiology 198: 523-529, 1996.

19 - BRADLEY WR \& QUENCER RM. Hydrocephalus, atrophy and CSF flow. In: DTARK DD \& BRADLEY WG JR, eds. Magnetic ressonance image. 2th. ed. St Louis, Mosby/Year Book, v.1, chap 28, 1992.

20 - WIKKELSO C \& ANDERSSON K. The clinical effects of lumbar puncture in normal pressure hydrocephalus. J Neurol Neurosurg Psychiatry 45: 64-69, 1982.

21 - BRADLEY WG; WHITTEMORE AR \& JINKINS JR. Marked cerebrospinal fluid void: Indicator os sucdcessful shunt in particular with suspected normal pressure hydrocephalus. Radiology 165: 611-616, 1987

22 - HUGHES CP; HOUSH TJ \& BENNET F. Adult idiophatic communicating hydrocephalus with and without shunting. $\mathbf{J}$ Neurol Neurosurg Psychiatry 41: 961-971, 1978.

23 - BORGENSEN SE. The predictive value of conductance to outflow of CSF in normal pressure hydrocephalus. Brain 105: 65-86, 1982.

Recebido para publicação em 26/01/2000.

Aprovado para publicação em 19/07/2000 